Thrust imparted by a stepped-diameter magnetic nozzle rf plasma thruster

Kazunori Takahashi, Yoshinori Takao, and Akira Ando

Citation: Appl. Phys. Lett. 113, 034101 (2018); doi: 10.1063/1.5041034

View online: https://doi.org/10.1063/1.5041034

View Table of Contents: http://aip.scitation.org/toc/apl/113/3

Published by the American Institute of Physics

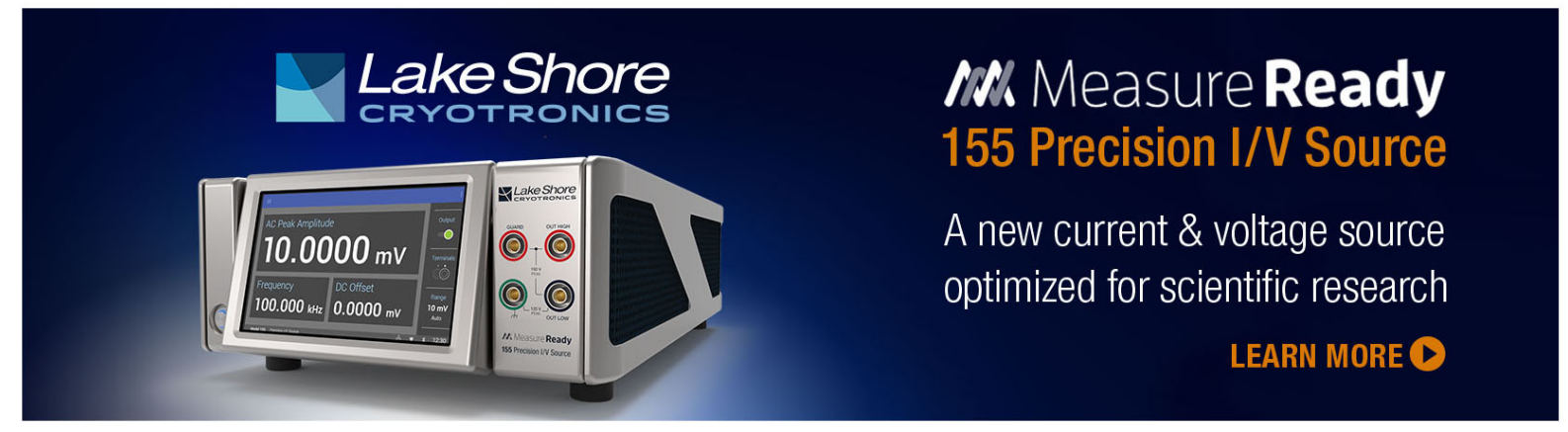




\title{
Thrust imparted by a stepped-diameter magnetic nozzle rf plasma thruster
}

\author{
Kazunori Takahashi, ${ }^{1, a)}$ Yoshinori Takao, ${ }^{2}$ and Akira Ando ${ }^{1}$ \\ ${ }^{1}$ Department of Electrical Engineering, Tohoku University, Sendai 980-8579, Japan \\ ${ }^{2}$ Division of Systems Research, Yokohama National University, Yokohama 240-8501, Japan
}

(Received 23 May 2018; accepted 3 July 2018; published online 17 July 2018)

\begin{abstract}
A stepped-diameter source tube is employed on a magnetic nozzle radiofrequency plasma thruster, where the magnetic field lines intersecting the wall near the antenna is separated from the radial wall near the thruster exit. The thruster is operated at a rf power up to $5 \mathrm{~kW}$. Comparison with the results for the cylindrical source tube shows the increase in the thrust by about $15 \%-20 \%$ for the stepped-diameter case in spite of the lower plasma density near the nozzle entrance, resulting in the lower electron-diamagnetic-induced thrust component. These indirectly show that the axial momentum lost to the radial wall is inhibited by separating the plasma flow from the radial source wall. Published by AIP Publishing. https://doi.org/10.1063/1.5041034
\end{abstract}

Electric propulsion devices utilizing external ion accelerations, e.g., ion gridded thrusters and hall thrusters, have been successfully developed for the last several decades and practically used for space propulsion, ${ }^{1}$ where propellant gas is ionized and anisotropically accelerated by dc electric fields; hence, the electrodes have to be exposed to plasmas or the discharge wall is exposed to the accelerated plasma. Ion sputtering and thermal load significantly affect the electrode and wall damage and the resultant lifetime of the propulsion device. One of the candidates to overcome the problem relating to the electrode lifetime is an electrodeless plasma thrusters utilizing a radiofrequency (rf) plasma production/heating and a spontaneous plasma acceleration in magnetic nozzles, e.g., a variable specific impulse plasma rocket (VASIMR), ${ }^{2}$ a helicon/rf plasma thruster, ${ }^{3-5}$ and an electron cyclotron resonance plasma thruster. ${ }^{6}$

Over the past decade, the physical mechanisms of the thrust generation in the magnetic nozzle plasma thrusters have been investigated by theoretical models, ${ }^{7-9}$ numerical calculations, ${ }^{10}$ and laboratory experiments. ${ }^{11-14}$ A number of experiments have shown spontaneous ion accelerations due to the formation of a current-free double layer and an ambipolar electric field in the magnetically expanding plasmas; ${ }^{15-18}$ subsequent Fruchtman's one-dimensional model has shown that the spontaneous electric field does not impart a momentum to the plasma, while the axial plasma momentum increases along the magnetic nozzle, ${ }^{7}$ which was followed by some two-dimensional models. ${ }^{19,20}$ Individual measurements of the force components exerted to the source tube and the magnetic fields have confirmed the role of the magnetic nozzle, ${ }^{21}$ where the model analyzed by Takahashi et al. has shown that the thrust increased by the magnetic nozzle corresponds to a Lorentz force due to an electron diamagnetic drift current and a radial magnetic field, which has been further investigated more recently in detail. ${ }^{22}$ Further downstream of the magnetic nozzle, the diamagnetic plasma flow state diverging the magnetic nozzle transits to that stretching the magnetic nozzle. ${ }^{23}$ Charles et al. have tested a conical source tube operated at the rf power less than $1 \mathrm{~kW}$;

a)kazunori@ecei.tohoku.ac.jp then their analysis includes the pressure term exerted to the gradually extended source tube, while the presence of a nonidentified thrust component has been implied. ${ }^{24}$ Subsequent individual measurements of the force exerted to the cylindrical radial wall demonstrates the presence of significant loss of the axial momentum to the radial wall, ${ }^{25}$ which seems to be enhanced by a neutral depletion effect occurring for the high rf power above a few $\mathrm{kW}^{26}$ and has been neglected in the previous models. ${ }^{27,28}$ The energy and momentum loss to the radial wall is very important to improve the thruster performance as discussed in Ref. 9. As described earlier, the magnetic nozzle plasma thruster seems to include many aspects of physics, both inside the source and in the magnetic nozzle; the inhibition of the loss to the radial wall is one of challenging topics to improve the performance.

Here, the stepped-diameter source tube combined with the magnetic nozzle is designed to verify the inhibition of the axial momentum lost to the radial source wall and the thrust of the magnetic nozzle rf plasma thruster having the stepped-diameter source tube is directly measured. In comparison of the thrusts between the cylindrical and steppeddiameter source tubes, an increase in the thrust by 15\%-20\% for the latter case is obtained, while the plasma density downstream of the thruster exit and the resultant thrust component induced by the magnetic nozzle are lower than those for the cylindrical case. These results imply the inhibition of the loss of the axial momentum to the radial wall by separating the radial wall from the plasma.

The experiment is performed in a 1-m-diameter and 2$\mathrm{m}$-long vacuum chamber evacuated by three turbomolecular pumping systems, resulting in a total effective pumping speed of $\sim 4200 \mathrm{~L} / \mathrm{s}$ for argon. A helicon plasma thruster (described later in detail) is attached to a pendulum thrust balance immersed in vacuum. A thruster displacement induced by a plasma production is measured by a high resolution laser sensor, giving the absolute value of the thrust force $T_{\text {total }}$ induced by the plasma production via multiplying a calibration coefficient, which is obtained before pumping down and confirmed after venting the chamber. ${ }^{26}$

Figure 1(a) shows a schematic diagram of the thruster consisting of a source tube, a solenoid, a back plate having a 
(a)

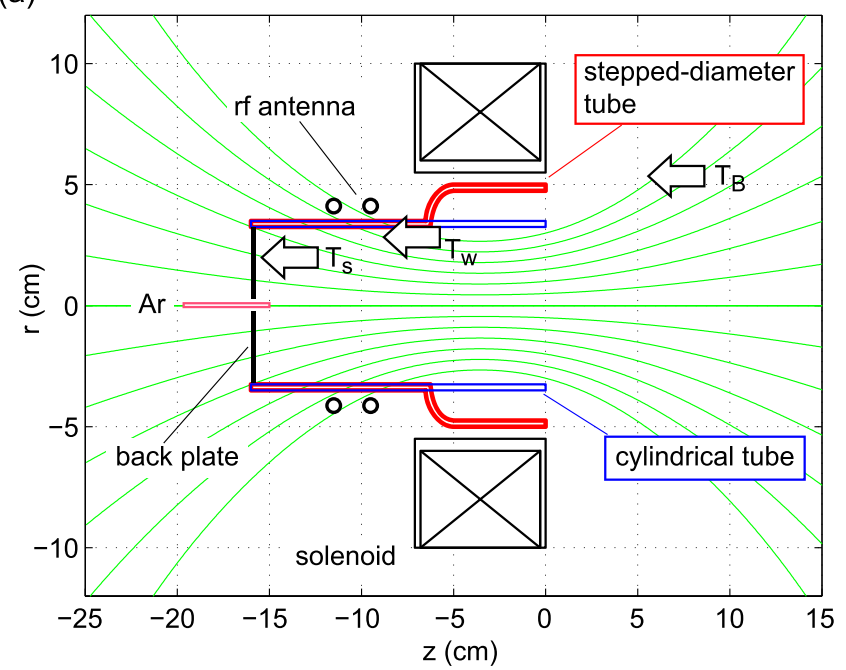

(b)

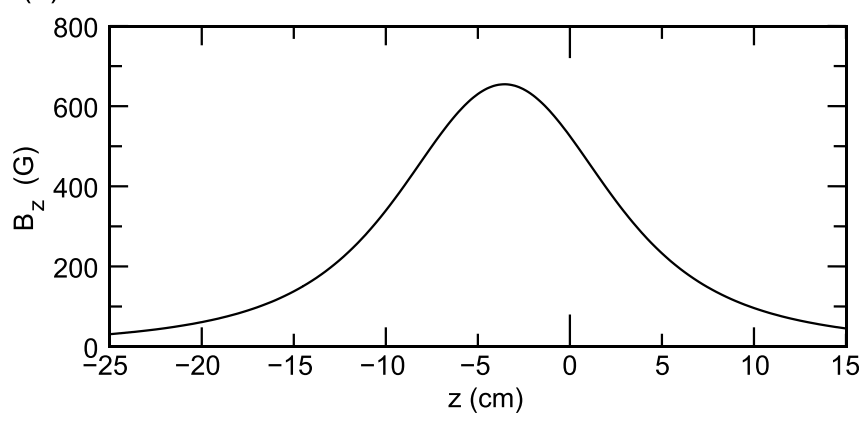

FIG. 1. (a) Schematic diagram of the magnetic nozzle rf plasma thruster, together with the calculated magnetic field lines. (b) Axial profile of the magnetic field strength on axis for $I_{B}=10 \mathrm{~A}$.

small center hole, and a water-cooled double-turn rf loop antenna shielded by a thick ceramic structure and a grounded metallic structure. ${ }^{29}$ The block arrows labelled as $T_{s}, T_{w}$, and $T_{B}$ are the force components exerted to the source back plate, to the radial source wall, and to the magnetic field lines, and the detailed explanation is described later in Eqs. (1)-(3). Argon gas is introduced from the back plate hole via a 2mm-outer-diameter ceramic tube, and the gas flow rate is maintained at $70 \mathrm{sccm}$ (corresponding to the mass flow rate of $2.1 \mathrm{mg} / \mathrm{s}$ ), resulting in the pressure at the chamber side port of $\sim 0.2$ mTorr. A dc current of $I_{B}=10 \mathrm{~A}$ is supplied to the solenoid to provide a magnetic field shown in Fig. 1(a) (magnetic field lines) and Fig. 1(b) (magnetic field strength on axis). The rf antenna is powered by a $13.56 \mathrm{MHz}$ rf generator via an impedance matching box located outside the chamber. Either of two different shape source tubes is employed here as shown in Fig. 1(a): one is a $65-\mathrm{mm}$-inner-diameter and 70-mm-outer-diameter cylindrical glass tube used previously (blue lines) and the other is a stepped-diameter $(65 \mathrm{~mm}-95 \mathrm{~mm}$ inner diameters and $70 \mathrm{~mm}-100 \mathrm{~mm}$ outer diameters) glass tube (red lines). The magnetic field lines intersecting the radial wall near the rf antenna is separated from the radial wall near the thruster exit for the stepped-diameter case, while the radial wall near the thruster exit is close to the field lines for the cylindrical case as seen in Fig. 1(a).

A planar Langmuir probe having a detector surface facing the direction perpendicular to the magnetic field lines (corresponding to the top side of Fig. 1) and having a
L-shape shaft is mounted on a movable motor stage immersed in vacuum (not shown in Fig. 1). The radial measurement can be approximately performed by rotating the shaft, and the radial position is obtained by a simple geometric calculation. The spatial profile of the ion saturation current $I_{i s}$ of the negatively biased Langmuir probe is measured for discussing underlying physics qualitatively. It should be mentioned that the ion collection surface facing perpendicular to the magnetic field lines allows to minimize the effect of the axial ion flow on the ion saturation current. The electron temperature measured at $z=-1 \mathrm{~cm}$ for both the source tubes are about $5 \mathrm{eV}$. Since the ion saturation current is proportional to the plasma density and the square root of the electron temperature, it can be used to discuss the plasma density for the roughly unchanged electron temperature obtained in experiment, where the change of the electron temperature is only $1-2 \mathrm{eV}$ for the present experimental parameters in the theoretical prediction with a global model. ${ }^{5}$

Figure 2 shows the directly measured thrusts $T_{\text {total }}$ for the cylindrical (filled circles) and the stepped-diameter (open squares) source tubes as functions of the rf power $P_{r f}$. As well as the previous studies, the thrust increases with an increase in the rf power for both the cases. ${ }^{26}$ The increase of $\sim 15 \%-20 \%$ in the thrust is obtained for the steppeddiameter source case, compared with that for the cylindrical case. Hence, it is demonstrated that the thruster performance is improvable by using the stepped-diameter shape of the source tube.

The axial measurement of the ion saturation current $I_{i s}$ in Fig. 3 shows the similar plasma density near the rf antenna $(z=-11.5 \mathrm{~cm})$ for both the cases, while the higher plasma density is detected for the cylindrical case between the antenna and the thruster exit, and over the axial length of about $\sim 15 \mathrm{~cm}$ downstream of the thruster exit. This difference in the density profile is expected to be originated from the geometric expansion of the neutrals in the steppeddiameter source, lowering the neutral density and reducing the ionization process near the thrust exit. The twodimensional $r-z$ profiles of the ion saturation current $I_{i s}$ are also taken by the Langmuir probe as shown in Fig. 4 and the

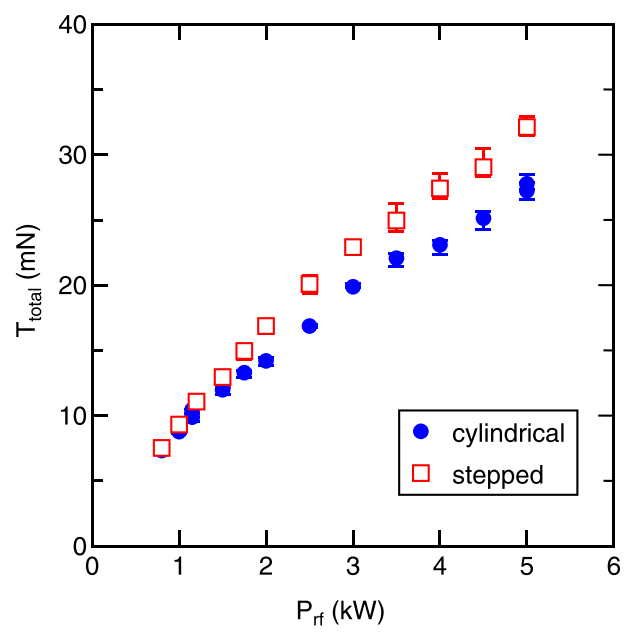

FIG. 2. Directly measured thrust $T_{\text {total }}$ of the magnetic nozzle rf plasma thruster having the cylindrical (filled squares) and stepped-diameter (open squares) source tubes, as functions of the rf power. 


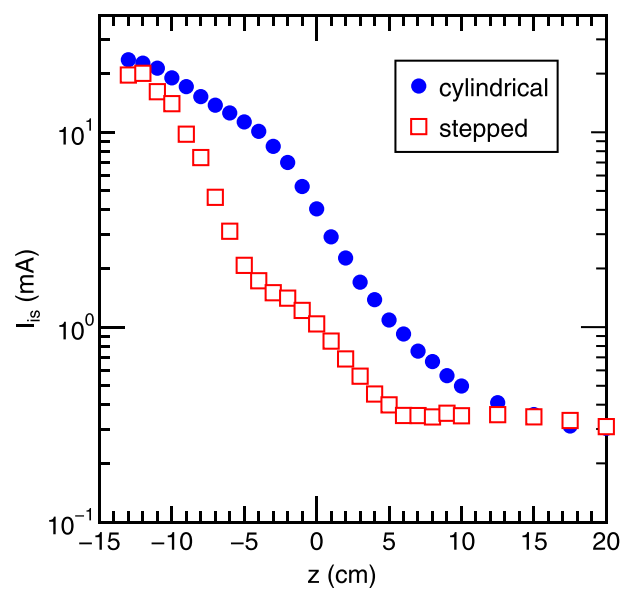

FIG. 3. The axial profiles of the ion saturation current $I_{i s}$ on axis with the cylindrical (filled circles) and stepped-diameter (open squares) source tubes, for the rf power of $P_{r f}=5 \mathrm{~kW}$.

representative radial profiles taken at $z=7 \mathrm{~cm}$ are shown in Fig. 5. The value of the ion saturation current downstream of the thruster exit for the cylindrical case is found to be larger than that for the stepped-diameter case over the radial region of the measurement as seen in Figs. 4 and 5. The radial location of the peripheral peak of the ion saturation current is similar for both the case. This results from the generation of the high temperature electrons near the rf antenna surrounding the 70-mm-outer diameter region, as presented previously in the cylindrical source, ${ }^{30-33}$ implying that the plasma generation in the present experiment mainly occurs in the 70-mm-diameter region even for the stepped-diameter case.

According to the previous theories, the total thrust $T_{\text {total }}$ is given by the sum of the forces exerted to the source back wall $T_{s}$, to the radial wall $T_{w}$, and to the magnetic field $T_{B}$, i.e., $T_{\text {total }}=T_{s}+T_{w}+T_{B}$, which is sketched in Fig. 1(a) and can be written as $8,13,25$

$$
\begin{gathered}
T_{s}=\left\langle p_{e s}\right\rangle A_{s}, \\
T_{w}=-\int_{S_{s}} \Gamma_{r} M_{z} d S, \\
T_{B}=-\int_{0}^{z} \frac{\left\langle p_{e}\right\rangle A}{B_{z}} \frac{\partial B_{z}}{\partial z^{\prime}} d z^{\prime},
\end{gathered}
$$

where $\left\langle p_{e s}\right\rangle, A_{s}, \Gamma_{r}, M_{z}, S_{s},\left\langle p_{e}\right\rangle$, and $A$ are the radiallyaveraged electron pressure at the maximum pressure position inside the source, the cross section of the source, the ion flux lost to the radial wall, the axial momentum of the ion lost to the wall, the surface area of the radial inner wall, the radially averaged electron pressure at $z$, and the plasma cross section at $z$, respectively. The previous experiment has detected the "negative" value of $T_{w}$ corresponding to the loss of the axial momentum to the radial wall as already mentioned. ${ }^{25}$

Total axial momentum of the plasma is generally given by the sum of the static electron pressure and the ion dynamic momentum when neglecting the ion temperature and the electron inertia. At the axial position giving a maximum pressure and a maximum potential, the total momentum is given only by the electron pressure due to the negligible ion velocity. Between the maximum potential position and the wall and near the back wall, which the $T_{s}$ force is exerted on, the
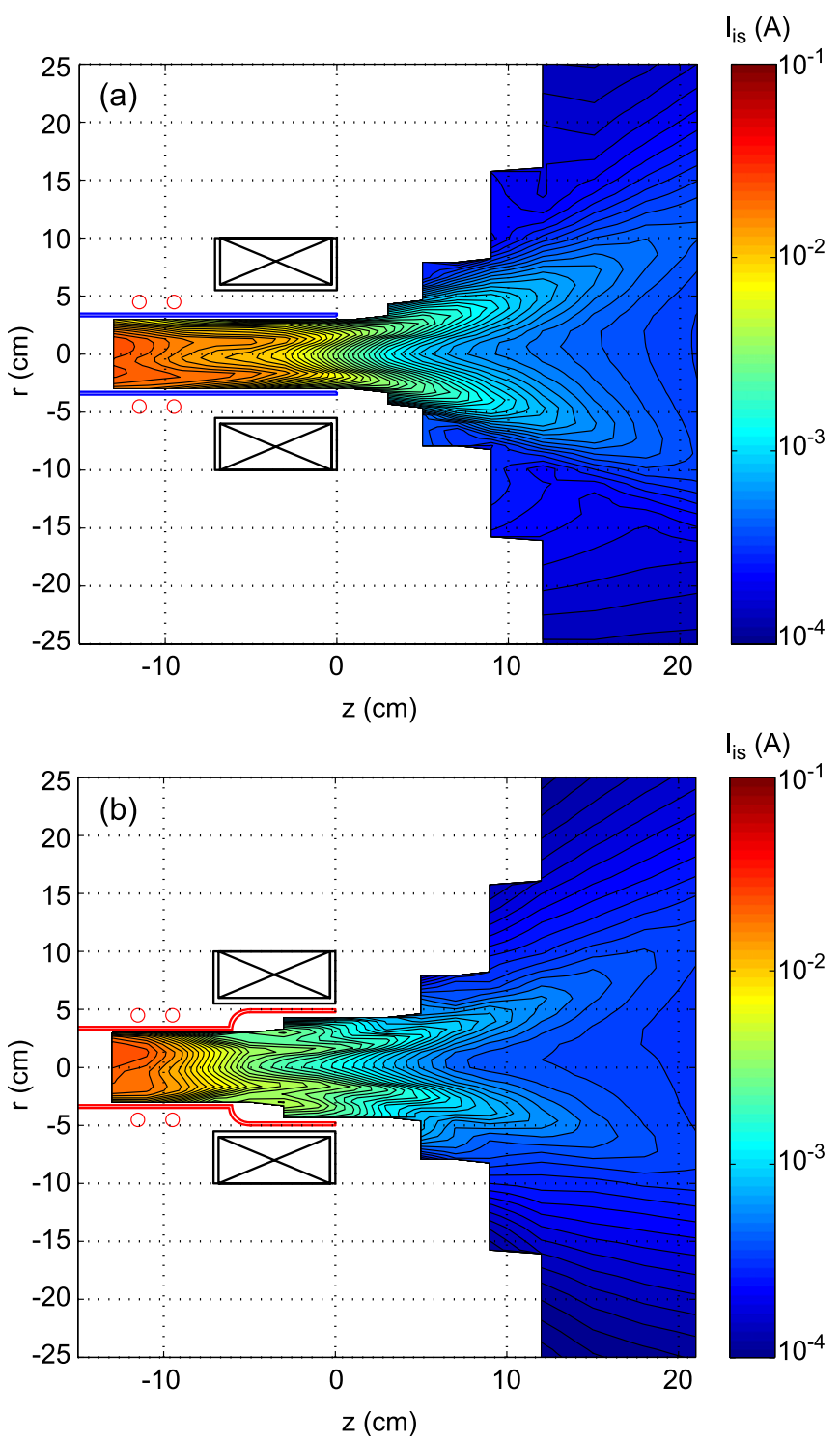

FIG. 4. Two-dimensional $(r-z)$ profiles of the ion saturation current $I_{i s}$ with the (a) cylindrical and (b) stepped-diameter source tube, for $P_{r f}=5 \mathrm{~kW}$.

ambipolar and sheath electric fields reflecting the electrons and accelerating the ions are spontaneously formed. Via the ion acceleration by the ambipolar and sheath electric fields, the electron pressure is converted into the ion dynamic momentum and the momentum is transferred to the back wall. This total momentum flux corresponds to the maximum electron pressure as being understood by analyzing the electron and ion momentum equations, which has been shown previously in $1-\mathrm{D}^{7}$ and 2-D models. ${ }^{34}$ The values of $T_{s}$ for $P_{r f}=5 \mathrm{~kW}$ calculated from the density profiles at the maximum pressure position of $z=-13 \mathrm{~cm}$ (Fig. 4) and the electron temperature of $\sim 5 \mathrm{eV}$ measured at $z=-1 \mathrm{~cm}$ are $19.1 \pm 3.1 \mathrm{mN}$ and $18.2 \pm 2.8 \mathrm{mN}$ for the cylindrical and stepped-diameter cases, respectively; no clear change in $T_{s}$ by the shape of the source tube is identified here due to the similar density inside the source. The stepped-diameter source also has the additional axial cross section at the connecting part $(z \sim-6 \mathrm{~cm})$ of the two different diameter glass tubes. Since the ion current is much smaller than that upstream of the source $(z \sim-13 \mathrm{~cm})$, the electron pressure force to the additional cross section is considered not to be significant. 


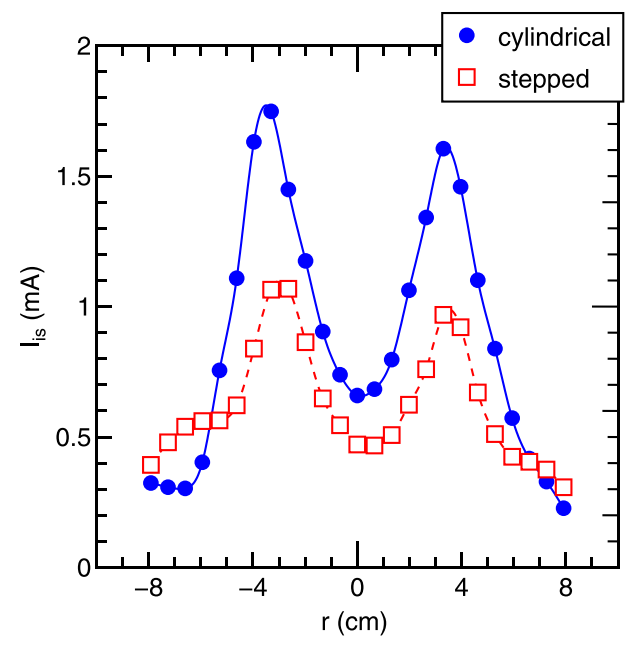

FIG. 5. Radial profile of the ion saturation current $I_{i s}$ with the cylindrical (filled circles) and stepped-diameter (open squares) source tubes, for the $\mathrm{rf}$ power of $P_{r f}=5 \mathrm{~kW}$. The solid and dotted lines are added as visual guides.

It was difficult to calculate Eqs. (2) and (3) from the experimental data due to difficulty of the measurement of $\Gamma_{r}$ and $M_{z}$ near the wall and due to difficulty of determining a radial integration range of the pressure, respectively; these two terms are very briefly and qualitatively discussed here. One of the previous experiments has shown that the thrust induced by the magnetic nozzle $\left(T_{B}\right)$ is generated over the 10-20 cm downstream of the thruster exit, ${ }^{22}$ where the $T_{B}$ term includes the volume integration in the magnetic nozzle, i.e., both the axial integration and the radially integrated term $\left\langle p_{e}\right\rangle A$ as shown in Eq. (3). The stepped-diameter source has a larger cross section of the thruster exit than the cylindrical case, while the spatial extension and the resultant plasma radius in the magnetic nozzle are very similar to that for the cylindrical case as in Figs. 4 and 5, originating from the major plasma production in the 65 -mm-diameter source region where the antenna is wound as discussed before. Therefore, the similar effective cross section can be considered in Eq. (3). In this region, the density for the cylindrical source case is higher than that for the stepped diameter case both along the axis as in Fig. 3 and over the radial position of the measurement as in Figs. 4 and 5, indicating a larger $T_{B}$ force generation as understood from Eq. (3) for the cylindrical case. However, the actually measured total thrust for the cylindrical case is smaller than that for the stepped-diameter case (Fig. 2), despite the similar value of $T_{s}$ to and the larger value of $T_{B}$ than the stepped-diameter case as discussed above. Hence, it is considered that the loss of the axial momentum to the wall (corresponding to the negative force of $T_{w}$ ) for the cylindrical case is more significant than that for the stepped-diameter case. In other words, these results show that the loss of the axial momentum to the radial wall (negative $T_{w}$ ) can be reduced by using the stepped-diameter source tube and resultantly separating the plasma from the radial source wall.

In summary, the direct thrust measurement of the magnetic nozzle rf plasma thruster having the stepped-diameter source tube shows the increase in the thrust by about
$15 \%-20 \%$, compared with the cylindrical case. The density inside the source tube is similar for both the case and the lower plasma density near the thruster exit (near the entrance of the magnetic nozzle) is detected for the stepped-diameter source case. This indicates the similar electron pressure force to the back wall and the small electron-diamagnetic-induced thrust for the stepped-diameter source tube. These results show that the axial momentum lost to the radial source wall is inhibited by having the stepped-diameter source tube.

This work was partially supported by JSPS KAKENHI Grant No. JP16H04084, Research Foundation for the Electrotechnology of Chubu, the Murata Science Foundation, and JAXA.

${ }^{1}$ S. Mazouffre, Plasma Sources Sci. Technol. 25, 033002 (2016) and references therein.

${ }^{2}$ B. W. Longmier, L. D. Cassady, M. G. Ballenger, M. D. Carter, F. R. Chang-Díaz, T. W. Glover, A. V. Ilin, G. E. McCaskill, C. S. Olsen, J. P. Squire, and E. A. Bering III, J. Propul. Power 27, 915 (2011).

${ }^{3}$ C. Charles, J. Phys. D: Appl. Phys. 42, 163001 (2009).

${ }^{4}$ L. T. Williams and M. L. R. Walker, J. Propul. Power 29, 520 (2013).

${ }^{5}$ K. Takahashi, A. Komuro, and A. Ando, Plasma Sources Sci. Technol. 24, 055004 (2015).

${ }^{6}$ F. Cannat, T. Lafleur, J. Jarrige, P. Chabert, P.-Q. Elias, and D. Packan, Phys. Plasmas 22, 053503 (2015).

${ }^{7}$ A. Fruchtman, Phys. Rev. Lett. 96, 065002 (2006).

${ }^{8}$ A. Fruchtman, K. Takahashi, C. Charles, and R. W. Boswell, Phys. Plasmas 19, 033507 (2012).

${ }^{9}$ T. Lafleur, Phys. Plasmas 21, 043507 (2014).

${ }^{10}$ Y. Takao and K. Takahashi, Phys. Plasmas 22, 113509 (2015).

${ }^{11}$ K. Takahashi, T. Lafleur, C. Charles, P. Alexander, R. W. Boswell, M. Perren, R. Laine, S. Pottinger, V. Lappas, T. Harle, and D. Lamprou, Appl. Phys. Lett. 98, 141503 (2011).

${ }^{12}$ S. Pottinger, V. Lappas, C. Charles, and R. Boswell, J. Phys. D: Appl. Phys. 44, 235201 (2011).

${ }^{13}$ K. Takahashi, C. Charles, and R. W. Boswell, Phys. Rev. Lett. 110, 195003 (2013).

${ }^{14}$ J. M. Little and E. Y. Choueiri, IEEE Trans. Plasma Sci. 43, 277 (2015).

${ }^{15}$ C. Charles, Plasma Sources Sci. Technol. 16, R1 (2007).

${ }^{16} \mathrm{~K}$. Takahashi and T. Fujiwara, Appl. Phys. Lett. 94, 061502 (2009).

${ }^{17}$ M. Wiebold, Y. Sung, and J. E. Scharer, Phys. Plasmas 18, 063501 (2011).

${ }^{18}$ B. B. Sahu, R. D. Tarey, and A. Ganguli, Phys. Plasmas 21, 023504 (2014).

${ }^{19}$ E. Ahedo and M. Merino, Phys. Plasmas 17, 073501 (2010).

${ }^{20}$ K. Takahashi, T. Lafleur, C. Charles, P. Alexander, and R. W. Boswell, Phys. Plasmas 19, 083509 (2012).

${ }^{21}$ K. Takahashi, T. Lafleur, C. Charles, P. Alexander, and R. W. Boswell, Phys. Rev. Lett. 107, 235001 (2011).

${ }^{22}$ K. Takahashi, A. Chiba, A. Komuro, and A. Ando, Plasma Sources Sci. Technol. 25, 055011 (2016).

${ }^{23}$ K. Takahashi and A. Ando, Phys. Rev. Lett. 118, 225002 (2017).

${ }^{24}$ C. Charles, K. Takahashi, and R. W. Boswell, Appl. Phys. Lett. 100, 113504 (2012).

${ }^{25}$ K. Takahashi, A. Chiba, A. Komuro, and A. Ando, Phys. Rev. Lett. 114, 195001 (2015).

${ }^{26}$ K. Takahashi, Y. Takao, and A. Ando, Appl. Phys. Lett. 109, 194101 (2016).

${ }^{27}$ A. Fruchtman, IEEE Trans. Plasma Sci. 36, 403 (2008).

${ }^{28}$ E. Ahedo and J. Navarro-Cavallé, Phys. Plasmas 20, 043512 (2013).

${ }^{29}$ K. Takahashi, Rev. Sci. Instrum. 83, 083508 (2012).

${ }^{30} \mathrm{~K}$. Takahashi, C. Charles, R. Boswell, W. Cox, and R. Hatakeyama, Appl. Phys. Lett. 94, 191503 (2009).

${ }^{31}$ C. Charles, Appl. Phys. Lett. 96, 051502 (2010).

${ }^{32}$ S. K. Saha, S. Chowdhury, M. S. Janaki, A. Ghosh, A. K. Hui, and S. Raychaudhuri, Phys. Plasmas 21, 043502 (2014).

${ }^{33}$ K. Takahashi, H. Akahoshi, C. Charles, R. W. Boswell, and A. Ando, Phys. Plasmas 24, 084503 (2017).

${ }^{34}$ T. Lafleur, K. Takahashi, C. Charles, and R. W. Boswell, Phys. Plasmas 18, 080701 (2011). 